\title{
Imprints of Spinning Particles on Primordial Cosmological Perturbations
}

\author{
Gabriele Franciolinia* $^{a}$, Alex Kehagias ${ }^{\mathrm{b} \dagger}$ and Antonio Riotto ${ }^{\mathrm{a}} \ddagger$ \\ ${ }^{a}$ Department of Theoretical Physics and Center for Astroparticle Physics (CAP) \\ 24 quai E. Ansermet, CH-1211 Geneva 4, Switzerland \\ ${ }^{\mathrm{b}}$ Physics Division, National Technical University of Athens, 15780 Zografou Campus, Athens, Greece
}

\begin{abstract}
If there exist higher-spin particles during inflation which are light compared to the Hubble rate, they may leave distinct statistical anisotropic imprints on the correlators involving scalar and graviton fluctuations. We characterise such signatures using the $\mathrm{dS} / \mathrm{CFT}_{3}$ correspondence and the operator product expansion techniques. In particular, we obtain generic results for the case of partially massless higher-spin states.
\end{abstract}

\section{Introduction}

Up to now, the most robust and successful mechanism to explain the primordial seeds for the cosmic microwave background anisotropies and the large-scale structure we observe in the universe is inflation [1], that is a (quasi-)de Sitter period when the physical space expands almost exponentially and quantum fluctuations initially at microscopic scales are stretched to macroscopical scales. After horizon re-entry, they initiate the phenomenon of gravitational instability giving rise to the structures of the universe.

From the high energy point of view, inflation is an appealing playground as it may happen at energies much larger than the electroweak scale and thus provide the most powerful collider to test physics at high energy [2-4]. For instance, cosmological correlators of the comoving curvature perturbation may be non-gaussian and originated from the exchange of massive higher-spin fields [4-7]. This generates some hope to learn something about their masses and spins.

A step towards the general characterisation of the cosmological perturbations generated during a de Sitter epoch has been the formulation of the so-called $\mathrm{dS} / \mathrm{CFT}_{3}$ correspondence [8]. During a period of exact exponentially expansion, the isometries of the corresponding dS spacetime form a $\mathrm{SO}(1,4)$ group which acts as the conformal group of a $\mathrm{CFT}_{3}$ on $\mathbb{R}^{3}$ and on the super-Hubble perturbations.

Technically, a four-dimensional field $A_{\mu_{1} \cdots \mu_{s}}(\vec{x}, \tau)$ with mass $m$ and spin $s$ evolves such that $A_{i_{1} \cdots i_{s}}(\vec{x}, \tau)=(-\tau)^{\Delta-s} A_{i_{1} \cdots i_{s}}(\vec{x})$ when approaching the boundary $\tau=0$ ( $\tau$ is the conformal time)

\footnotetext{
*gabriele.franciolini@unige.ch

†kehagias@central.ntua.gr

łantonio.riotto@unige.ch
} 
with

$$
\Delta=\frac{3}{2}-\sqrt{\left(s-\frac{1}{2}\right)^{2}-\frac{m^{2}}{H^{2}}},
$$

$H$ being the Hubble rate during inflation. The field $A_{i_{1} \cdots i_{s}}(\vec{x})$ behaves like a primary field with conformal weight $\Delta$ under the boundary conformal transformations. At this stage, it is interesting to point out that one can give two different interpretations of the $\mathrm{SO}(1,4)$ group. When gravitational fluctuations are excited, a case relevant for single-field models of inflation, $\mathrm{SO}(1,4)$ is identified with the three-dimensional conformal group at different constant time-slices; in the limit in which gravity is decoupled, holding when one is interested in spectator fields (such as in the curvaton model [9]), including higher-spin fields, $\mathrm{SO}(1,4)$ is a non-linearly realised symmetry of the action in de Sitter.

De Sitter isometries play an important role when dealing with massive spinning fields: the mass $m$ and the spin $s$ of a higher-spin field in de Sitter must respect the Higuchi bound [10] to avoid the lower helicity modes to become ghost-like

$$
m^{2}>s(s-1) H^{2},
$$

This inequality can be inferred from the $\mathrm{dS} / \mathrm{CFT}_{3}$ correspondence $[4,11,12]$ and implies that higherspin super-Hubble two-point correlators $\left\langle A_{i_{1} \cdots i_{s}} A^{i_{1} \cdots i_{s}}\right\rangle$ decay as $(-\tau)^{2 \Delta}$ with $\Delta>1$, as one can deduce from the relation (1.1). Higher-spin fields are therefore short-lived and their impact on cosmological observables is rather suppressed.

To the best of our knowledge there are two ways to evade the Higuchi bound. On one side, one can exploit partially massless higher-spin fields [13-17]. Indeed, for some particular values of their masses, some helicities of spinning states may acquire a vanishing conformal weight in de Sitter so that their fluctuations can be excited with a scale-invariant spectrum during inflation. From the point of view of the $\mathrm{dS} / \mathrm{CFT}_{3}$ correspondence, these states correspond to rank- $s$ symmetric boundary tensors which are partially conserved $[18,19]$.

On the other side, to obtain vanishing conformal weights for massive higher-spin fields, one can couple the higher-spin states to a preferred foliation of spacetime such that the quadratic action is not covariant. This phenomenon may take place by coupling the higher-spin fields to a suitable function of time (or better of the classical value $\phi_{0}$ of the inflaton field). For instance, for spin-1 fluctuations a modification of the kinetic term of the form $I\left(\phi_{0}\right) F_{\mu \nu}^{2}$ may lead to constant superHubble perturbations of the electric or magnetic fields [20-22] and the scalar and vector correlators can be determined exploiting the $\mathrm{dS} / \mathrm{CFT}_{3}$ correspondence [23]. An extension of such mechanism to higher-spin has been done in Ref. [24]. There suitable time-dependent functions of time coupled to the higher-spin fields have been identified in such a way that the lower helicity modes are prevented from becoming ghost-like, still allowing masses below the Higuchi bound and preserving the correct number of degrees of freedom for the higher-spin fields. These couplings give rise to enhanced symmetries and correlators decaying slower than what dictated by the Higuchi bound outside the Hubble radius.

When spinning degrees of freedom are quantum mechanically excited and remain constant on superHubble scales, they leave a distinctive statistical anisotropic signature on the cosmological correlators [25-28]. This happens because during the prolonged period of inflation an infrared background of the higher-spin field is generated with a magnitude of order of the square root of its variance. During the last 60 e-folds or so, when the scalar modes exit the Hubble radius at comoving wavenumbers 
corresponding to cosmologically relevant length scales, and taking our observed universe as a single realisation of the different ensemble ones [27], they live in a background which is slightly anisotropic due to such non-vanishing infrared vacuum expectation value of the spinning fields. The statistical anisotropy is distinctive since the angle structure depends on the spins. If observed, the induced anisotropies will deliver fundamental informations about the particle content at high energies.

The set-up we will be considering therefore is the following, see Fig. 1:

1. there exist spinning light degrees of freedom whose two-point correlators are approximately constant on super-Hubble scales; they provide a representation of de Sitter isometry group and coincide with those of the $\mathrm{CFT}_{3}$ on super-Hubble scales;

2. inflation lasts more than the canonical minimal $\sim 60$ e-folds to explain the features of our observed universe. In this way, in the last 60 e-folds or so there exists a vacuum expectation value of the higher-spin field which introduces preferred directions, breaking isotropy. Here it is important to notice that fluctuations with comoving wavenumber $k$ exit the Hubble radius after a single realisation of the first $\left(N-N_{k}\right)$ e-folds of inflation, where $N$ is the total number of e-folds and $N_{k}$ is the number of e-folds till the end of inflation when such modes exit. They are affected therefore by the value that the infrared higher-spin field assumes in that single realisation. The fact that the higher-spin state gets a vacuum expectation value is relevant to our considerations since it allows, in analogy with the spin-1 case, a mixing between scalars and higher-spin fields.

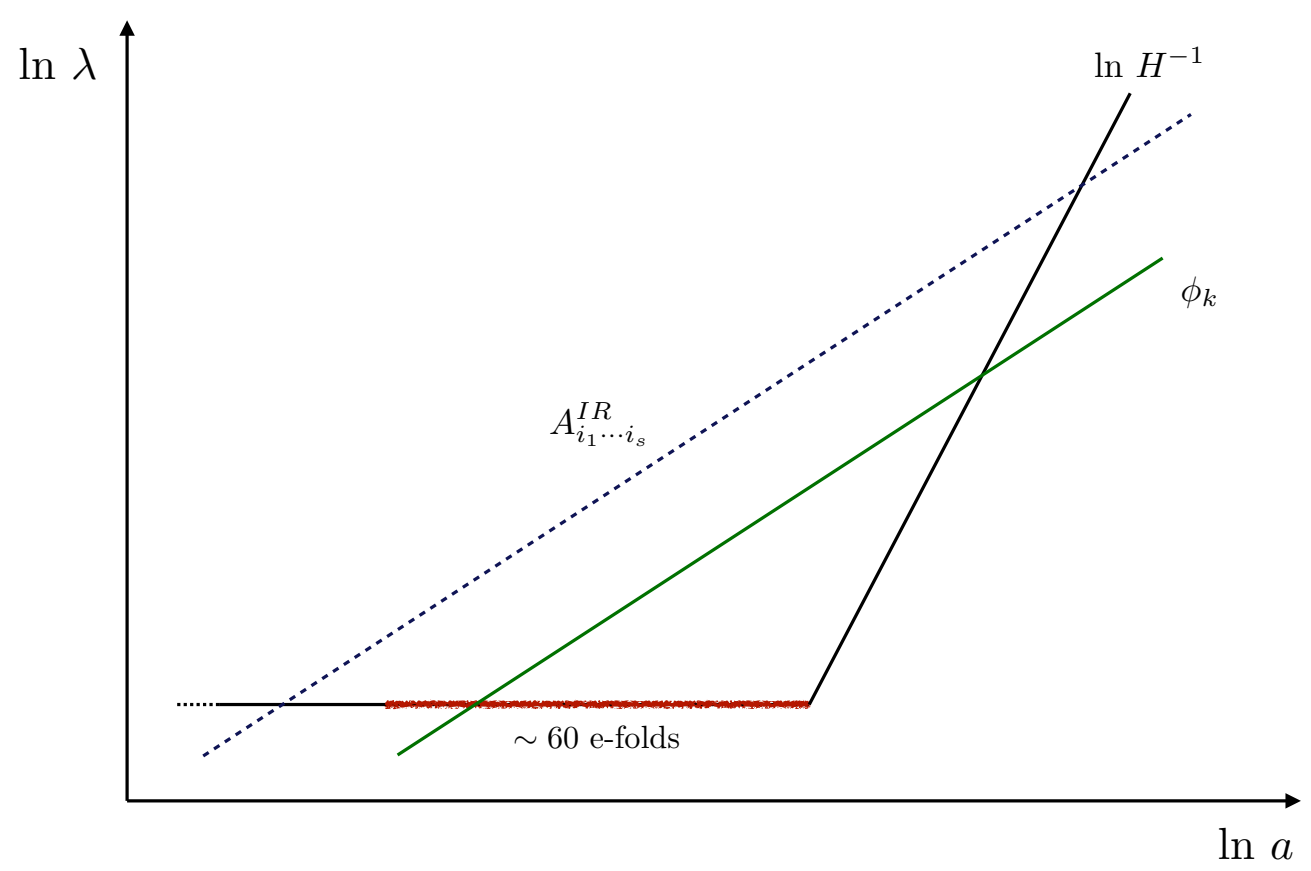

Figure 1: Scalar perturbations on cosmologically relevant scales leave the Hubble radius in the presence of a nonvanishing higher-spin infrared background.

The goal of this paper is to characterise the statistically anisotropic signals of the effectively massless higher-spin fields onto the power spectrum, the bispectrum and the trispectrum of the scalar pertur- 
bations from the point of view of the $\mathrm{dS} / \mathrm{CFT}_{3}$ correspondence when the higher-spin states acquire a vacuum expectation value.

We will follow and generalise the techniques developed by Cardy [29] to compute the anisotropic corrections to correlation functions in conformal systems where some components of the energy momentum tensor acquire non-zero vacuum expectation values.

A technical tool which will be useful in obtaining our findings will be the Operator Product Expansion (OPE), see for example Refs. [30,31]. We will do so by both analysing the case of the partially massless spinning states and extending the findings of Ref. [23] to the case of fields with spin larger than unity. Our technique will also allow us to easily reproduce the well-known results of the spin-1 case [27].

Since in the case of single-field models of inflation, the inflaton background is still invariant under a dilations plus a shift of the inflaton field, but not under special conformal transformations, rigorously our considerations will be valid only in the case in which scalar perturbations arise from multi-field models of inflation, e.g. through the curvaton mechanism, where the perturbations are induced by a spectator field $\phi(\tau, \vec{x})$ during inflation. In such a case gravity is decoupled and $\mathrm{SO}(1,4)$ is a nonlinearly realised symmetry of the action in de Sitter. In the single-field models of inflation the scalar fluctuations are sensitive to departures from the special conformal symmetry and we expect that a systematic breaking of such symmetries could lead to further constraints at leading order in the slow roll parameters ${ }^{1}$.

The paper is organised as follows. In section 2 we study the statistically anisotropic contributions to the scalar two-point correlator from spinning particles, devoting section 3 to the study of the threeand four-point correlators. Section 4 is devoted to the analysis restricted to the case of partially massless higher-spin states. Section 5 contains our conclusions.

\section{The statistically anisotropic two-point correlator from spinning particles}

In this section we wish to characterize the anisotropic contributions to the power spectrum of scalar fluctuations induced by the presence of constant super-Hubble modes of higher-spin fields.

\subsection{The spin-1 case}

Let us start with the known case of a spin-1 field $[23,27]$ which, thanks to a suitable coupling of the form $I\left(\phi_{0}\right) F_{\mu \nu}^{2}$, has vanishing scaling dimension.

From now on we call $\phi(\tau, \vec{x})$ the (spectator scalar) field which scales towards the boundary $\tau=0$ as $\phi(\tau, \vec{x})=(-\tau)^{\Delta_{\phi}} \phi(\vec{x})$. In this sense, we identify with $\phi(\vec{x})$ the scalar primary field with conformal weight $\Delta_{\phi} \simeq 0$. We also assume that the corresponding conformal weight of the spin-1 field on the boundary is close to zero. The two-point function $\left\langle\phi\left(\vec{x}_{1}\right) \phi\left(\vec{x}_{2}\right)\right\rangle$ can be worked out in the following

\footnotetext{
${ }^{1}$ The case of a single-field inflation and the role of partially massless higher-spin fields is discussed in Ref. [32]. We thank D. Baumann, G. Goon, H. Lee and G.L. Pimentel for sharing their draft with us.
} 
way. Let us perform an inversion with respect to the origin far from the points $\vec{x}_{1}$ and $\vec{x}_{2}$

$$
x_{1}^{i}=\frac{x_{1}^{i}}{x_{1}^{2}}, \quad x_{2}^{i i}=\frac{x_{2}^{i}}{x_{2}^{2}},
$$

such that the new points $x_{1}^{\prime}$ and $x_{2}^{\prime}$ are brought close to each other,

$$
\left\langle\phi\left(\vec{x}_{1}\right) \phi\left(\vec{x}_{2}\right)\right\rangle=x_{1}^{\prime 2 \Delta_{\phi}} x_{2}^{\prime 2 \Delta_{\phi}}\left\langle\phi\left(\vec{x}_{1}^{\prime}\right) \phi\left(\vec{x}_{2}^{\prime}\right)\right\rangle .
$$

Since now the points $x_{1}^{\prime}$ and $x_{2}^{\prime}$ are close, we can perform the OPE expansion on the two-point correlator on the right-hand side. We assume that the vector field is $A^{i}(\vec{x})$ on the boundary and that its two-point function is fixed by the symmetries, up to the an overall normalization. More importantly, we also assume that the spin-1 fields acquire a vacuum expectation value since during inflation the infrared long wavelength perturbations of the vector field accumulate and provide a classical background for a local observer.

The field $\phi(\vec{x})$ mixes with the scaling invariant operator $A_{i}(\vec{x})$ so that in the OPE expansion we expect terms of the form

$$
\phi\left(\vec{x}_{1}^{\prime}\right) \phi\left(\vec{x}_{2}^{\prime}\right) \simeq \frac{c_{\phi}}{{x^{\prime 2}}_{12}^{2 \Delta_{\phi}}}+c_{i}\left(x_{12}^{\prime}\right) A^{i}\left(\vec{x}_{2}^{\prime}\right)+c_{i j}\left(x_{12}^{\prime}\right) A^{i} A^{j}\left(\vec{x}_{2}^{\prime}\right)+\cdots,
$$

where we have adopted the common notation $x_{k m}^{i}=\left(x_{k}^{i}-x_{m}^{i}\right)$. Due to the gauge invariance symmetry associated to the gauge field $A_{i}(\vec{x})$, it is easy to show that the coefficients of the OPE expansion in Fourier space must satisfy the following relations ${ }^{2}$

$$
k^{i} c_{i}(k)=k^{i} c_{i j}(k)=0,
$$

from which we deduce the relations $c_{i}(k)=0$ and $c_{i j}(k)=c(k) \Pi_{i j}(k)$, where

$$
\Pi_{i j}(k)=\delta_{i j}-\hat{k}_{i} \hat{k}_{j}, \quad \hat{k}_{i}=\frac{k_{i}}{k} .
$$

Undoing now the inverse transformation to go back to the original coordinates and performing the Fourier transform respect to $\vec{x}_{12}$ we get

$$
\left\langle\phi_{\vec{k}} \phi_{-\vec{k}}\right\rangle^{\prime}=\frac{c_{\phi}}{k^{3}}+c(k)\left(\delta_{i j}-\hat{k}_{i} \hat{k}_{j}\right)\left\langle A^{i} A^{j}(0)\right\rangle+\cdots .
$$

The prime here indicates dropping the Dirac delta for momentum conservation and the factor $(2 \pi)^{3}$. At this stage we pause and provide two comments. First, the OPE, since it is a short-distance property of the theory, it is not affected by boundary conditions provided by the vacuum expectation value of the vector field. Secondly, the quantity $\left\langle A^{i} A^{j}(0)\right\rangle$ gets two contributions, one from a possible classical value pre-existing the start of inflation and another inevitable one generated from the beginning of inflation and caused by the accumulation of the infrared modes. It is seen by a local observer restricted on a finite Hubble volume as a background which breaks isotropy. Following Ref. [27] and taking our observed universe as a single realisation of the different ensemble ones, we can write

$$
\left\langle A^{i} A^{j}(0)\right\rangle=A_{0}^{2} n^{i} n^{j}
$$

\footnotetext{
${ }^{2}$ Of course one can start working directly with gauge-invariant fields, in the case at hand with the electric or the magnetic fields [23,27].
} 
where $\vec{n}$ identifies the preferred direction of such a single realisation. The typical value of $A_{0}$ is in such a case the square root of the variance of the vector field when a given wavenumber $k$ of the scalar fluctuations leave the comoving Hubble radius, that is $A_{0} \sim H \sqrt{N-N_{k}}$, where $N$ is the total number of e-folds of inflation and $N_{k}$ is the number of e-folds till the end of inflation when a given comoving wavelength $1 / k$ exists the comoving Hubble radius ${ }^{3}$.

In order to fix the coefficient $c(k)$ we use the Ward identity associated to the dilations (we take $\left.\Delta_{\phi} \simeq 0\right)$

$$
\left(3+k^{i} \partial_{k_{i}}\right)\left\langle\phi_{\vec{k}} \phi_{-\vec{k}}\right\rangle^{\prime}=0
$$

or

$$
3 c(k)+k \partial_{k} c(k)=0,
$$

which gives

$$
c(k)=\frac{c_{1}}{k^{3}} .
$$

Collecting these results we finally obtain the anisotropic contribution to the scalar power spectrum to be

$$
\left\langle\phi_{\vec{k}} \phi_{-\vec{k}}\right\rangle^{\prime}=\frac{c_{\phi}}{k^{3}}\left(1+c_{1} \sin ^{2}(\hat{k} \cdot \hat{n})+\cdots\right) .
$$

This angle dependence, obtained solely by symmetry arguments, nicely reproduces the one in Ref. [27].

\subsection{The higher-spin case}

One can now proceed similarly for the fields with spin larger than unity and conformal weight close to zero. Along the same lines, one can write the OPE as

$$
\phi_{\vec{k}} \phi_{-\vec{k}} \simeq \frac{c_{\phi}}{k^{3}}\left(1+c_{i_{1} \cdots i_{s} j_{1} \cdots j_{s}}(k) A^{i_{1} \cdots i_{s}} A^{j_{1} \cdots j_{s}}(0)+\cdots\right) .
$$

Due to an enhanced symmetry for special values of the parameters in the higher-spin field equations coupled to suitable functions of time [24], the coefficients $c_{i_{1} \cdots i_{s} j_{1} \cdots j_{s}}(k)$ satisfy the gauge invariance condition

$$
k^{i_{1}} c_{i_{1} \cdots i_{s} j_{1} \cdots j_{s}}(k)=\cdots=k^{i_{s}} c_{i_{1} \cdots i_{s} j_{1} \cdots j_{s}}(k)=0,
$$

and are symmetric and traceless in the first and second group of indices as well as in the interchange of the two groups of indices. Clearly, these coefficients are proportional to the spin helicity sums

$$
c_{i_{1} \cdots i_{s} j_{1} \cdots j_{s}}(k)=c(k) \sum_{\lambda} \epsilon_{i_{1} \cdots i_{s}}^{\lambda}(k) \epsilon_{j_{1} \cdots j_{s}}^{* \lambda}(k),
$$

where $\lambda$ are the helicities and the polarisation tensors $\epsilon_{i_{1} \cdots i_{s}}^{\lambda}$ are symmetric traceless and satisfy the relations

$$
k^{i_{1}} \epsilon_{i_{1} \cdots i_{s}}^{\lambda}=0, \quad \epsilon^{\lambda i_{1}}{ }_{i_{1} i_{3} \cdots i_{s}}=0 .
$$

\footnotetext{
${ }^{3}$ Since the associated energy density is of the order of $H^{4} N$, the bound $N<\left(M_{\mathrm{Pl}} / H\right)^{2}$ must be satisfied to avoid that the energy stored into the infrared modes exceeds the one driving inflation. Notice also that on the last 60 e-folds or so, the classical background evolution in time is suppressed by powers of $N_{k} / N \ll 1$ and therefore the background can be taken constant with time with good approximation.
} 
Polarisation tensors of higher-spin fields can be obtained generalising the notion of polarisation vectors introducing positive and negative energy wave functions

$$
\begin{aligned}
\epsilon_{\lambda}^{i_{1} \cdots i_{s}}(k) & =\sum_{\lambda_{1}, \cdots, \lambda_{s}= \pm 1} \delta_{\lambda_{1}+\cdots+\lambda_{s}, \lambda} \sqrt{\frac{2^{s}(s+\lambda) !(s-\lambda) !}{(2 s) ! \prod_{i=1}^{s}\left(1+\lambda_{i}\right) !\left(1-\lambda_{i}\right) !}} \prod_{j=1}^{s} \epsilon_{\lambda_{j}}^{i_{j}}(k), \\
\epsilon_{\lambda}^{* i_{1} \cdots i_{s}}(k) & =\sum_{\lambda_{1}, \cdots, \lambda_{s}= \pm 1} \delta_{\lambda_{1}+\cdots+\lambda_{s}, \lambda} \sqrt{\frac{2^{s}(s+\lambda) !(s-\lambda) !}{(2 s) ! \prod_{i=1}^{s}\left(1+\lambda_{i}\right) !\left(1-\lambda_{i}\right) !}} \prod_{j=1}^{s} \epsilon_{\lambda_{j}}^{* i_{j}}(k),
\end{aligned}
$$

where $\epsilon_{\lambda}^{i}$ and $\epsilon_{\lambda}^{* i}$ are positive and negative energy wave functions for a spin-1 field, with

$$
\epsilon_{\lambda}^{* i}=(-1)^{\lambda} \epsilon_{-\lambda}^{i}
$$

It is useful to define the projector tensor in $d$ dimensions as

$$
\Pi^{i_{1} \cdots i_{s} j_{1} \cdots j_{s}}(k) \equiv \sum_{\lambda} \epsilon_{\lambda}^{i_{1} \cdots i_{s}}(k) \epsilon_{\lambda}^{* j_{1} \cdots j_{s}}(k)
$$

It can be explicitly constructed by using the spin-1 projector tensor $\Pi^{i j}$

$$
\Pi^{i_{1} \cdots i_{s} j_{1} \cdots j_{s}}(k)=\left(\frac{1}{s !}\right) \sum_{P(i) P(j)}\left[\sum_{r=0}^{r \leq \frac{s}{2}} C(s, r) \Pi^{i_{1} i_{2}} \Pi^{j_{1} j_{2}} \cdots \Pi^{i_{2 r-1} i_{2 r}} \Pi^{j_{2 r-1} j_{2 r}} \prod_{n=2 r+1}^{s} \Pi^{i_{n} j_{n}}\right],
$$

where $P(i) P(j)$ stands for independent permutations of $i$ and $j$ sets of indices and, by defining a function $A(m, n)$ so that

$$
\begin{aligned}
A(m, n) & =\left(\begin{array}{c}
m \\
2
\end{array}\right)\left(\begin{array}{c}
m-2 \\
2
\end{array}\right) \cdots\left(\begin{array}{c}
m-2(n-1) \\
2
\end{array}\right), \\
A(m, n) & =0 \text { for } n<0, \quad A(m, n)=1 \text { for } n=0, \quad A(m, n)=0 \text { for } m<2 n .
\end{aligned}
$$

Thus, the coefficients in Eq. (2.19) are

$$
C(s, r)=-\left\{\frac{C(s, r-1) A(s, r-1) A(s-2, r-1)[s-2(r-1)] !}{A(s, r)(s-2 r) ![A(s, r)-A(s-2, r)+(d-2) A(s-2, r-1)]}\right\}, \quad C(s, 0)=1 .
$$

For instance, for spin-2 in three-dimensions we obtain (we of course sum only over the maximally transverse modes as lower helicity states decay on super-Hubble scales)

$$
\Pi_{i_{1} i_{2}}^{j_{1} j_{2}}=\frac{1}{2}\left(\Pi_{i_{1}}^{j_{1}} \Pi_{i_{2}}^{j_{2}}+\Pi_{i_{1}}^{j_{2}} \Pi_{i_{2}}^{j_{1}}\right)-\frac{1}{2} \Pi_{i_{1} i_{2}} \Pi^{j_{1} j_{2}}
$$

The coefficient of proportionality can be fixed as before using the Ward identity associated to the dilation symmetry. Thus, we have

$$
\left\langle\phi_{\vec{k}} \phi_{-\vec{k}}\right\rangle^{\prime}=\frac{c_{\phi}}{k^{3}}\left(1+c_{i_{1} \cdots i_{s} j_{1} \cdots j_{s}}(k)\left\langle A^{i_{1} \cdots i_{s}} A^{j_{1} \cdots j_{s}}(0)\right\rangle+\cdots\right) .
$$

We assume again that there is a background value for the higher-spin field which defines a set of $s$ vectors $n_{m}^{i}$ with $m=1, \cdots, s$ (not necessarily normalised to unity), such that

$$
\left\langle A^{i_{1} \cdots i_{s}}\right\rangle=\left[n_{1}^{\left(i_{1}\right.} \cdots n_{s}^{\left.i_{s}\right)}+\cdots\right],
$$


where the $\cdots$ denote extra terms constructed with Kronecker deltas in such a way to preserve the traceless constraint. In any case, these extra terms are irrelevant when contracted with the polarisation tensors. We then obtain

$$
\left\langle A^{i_{1} \cdots i_{s}} A^{j_{1} \cdots j_{s}}(0)\right\rangle=\left[n_{1}^{\left(i_{1}\right.} \cdots n_{s}^{\left.i_{s}\right)} n_{1}^{\left(j_{1}\right.} \cdots n_{s}^{\left.j_{s}\right)}+\cdots\right],
$$

The two-point correlator of the bulk field $\phi$ turns out to be

$$
\left\langle\phi_{\vec{k}} \phi_{-\vec{k}}\right\rangle^{\prime}=\frac{c_{\phi}}{k^{3}}\left(1+c_{s} \prod_{i=1}^{s} \sin ^{2}\left(\hat{k} \cdot \hat{n}_{i}\right)+\cdots\right) .
$$

Notice that, in the special case in which all the $\vec{n}_{m}$ are aligned along a common direction $\vec{n}$ and a subgroup $\mathrm{SO}(2)$ rotation symmetry is present, the background value for the higher-spin field is

$$
\left\langle A^{i_{1} \cdots i_{s}}\right\rangle=A_{0}\left[n^{i_{1}} \cdots n^{i_{s}}-\frac{1}{2 s-1}\left(\delta^{i_{1} i_{2}} n^{i_{3}} \cdots n^{i_{s}}+\text { symm. }\right)+\cdots\right]
$$

and the previous equation reduces to the one adopted in Ref. [28]

$$
\left\langle\phi_{\vec{k}} \phi_{-\vec{k}}\right\rangle^{\prime}=\frac{c_{\phi}}{k^{3}}\left(1+c_{s} \sin ^{2 s}(\hat{k} \cdot \hat{n})+\cdots\right) .
$$

From now on, to simplify the expressions, we will be assuming that the vectors $\vec{n}_{m}$ are aligned along a common direction $\vec{n}$.

\section{The statistically anisotropic three- and four-point cor- relator from spinning particles}

In this section we wish to characterise the anisotropic contributions to the three- and four-point correlators of scalar and tensor fluctuations induced by the presence of constant super-Hubble modes of higher-spin fields.

\subsection{The spin-1 case}

Let us again start with the known case of the spin-1 field [23,27]. The three-point function $\left\langle\phi\left(\vec{x}_{1}\right) \phi\left(\vec{x}_{2}\right) \phi\left(\vec{x}_{3}\right)\right\rangle$ can be worked out by performing an inversion with respect to a point $\vec{z}$ [29], see Fig. 2

$$
x^{i}=\frac{x^{i}-z^{i}}{|\vec{x}-\vec{z}|^{2}}
$$

If we take $\vec{z}$ in the vicinity of $\vec{x}_{3}$, than the new points $x_{1}^{\prime}$ and $x_{2}^{\prime}$ are brought close to each other and far from $x_{3}^{\prime}$. We therefore get

$$
\left\langle\phi\left(\vec{x}_{1}\right) \phi\left(\vec{x}_{2}\right) \phi\left(\vec{x}_{3}\right)\right\rangle=x_{1}^{\prime 2 \Delta_{\phi}} x_{2}^{\prime 2 \Delta_{\phi}} x_{3}^{\prime 2 \Delta_{\phi}}\left\langle\phi\left(\vec{x}_{1}^{\prime}\right) \phi\left(\vec{x}_{2}^{\prime}\right) \phi\left(\vec{x}_{3}^{\prime}\right)\right\rangle .
$$

This time we find it convenient to perform the OPE by expanding in powers of the scalar quantity $\mathcal{A}(\vec{x})=A_{0}^{i} A_{i}(\vec{x})$ 


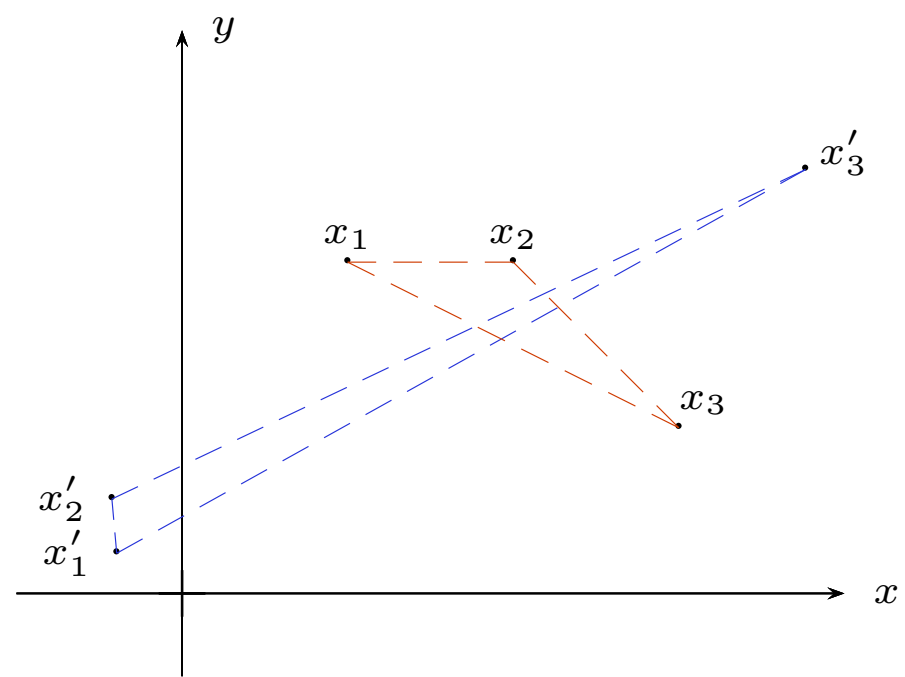

Figure 2: The inversion operation around a point $z$ close to $\vec{x}_{3}$.

$$
\phi\left(\vec{x}_{1}^{\prime}\right) \phi\left(\vec{x}_{2}^{\prime}\right) \simeq \frac{c_{\phi}}{{x^{\prime 2}}_{12}^{2 \Delta_{\phi}}}\left(1+c_{1} \mathcal{A}\left(\vec{x}_{2}^{\prime}\right)+c_{2} \mathcal{A}^{2}\left(\vec{x}_{2}^{\prime}\right)+\cdots\right) .
$$

From this expression we can deduce

$$
\left\langle\phi\left(\vec{x}_{1}^{\prime}\right) \phi\left(\vec{x}_{2}^{\prime}\right) \phi\left(\vec{x}_{3}^{\prime}\right)\right\rangle \simeq \frac{c_{2}}{{x^{\prime \prime}}_{12}^{2 \Delta_{\phi}}}\left\langle\mathcal{A}^{2}\left(\vec{x}_{2}^{\prime}\right) \phi\left(\vec{x}_{3}^{\prime}\right)\right\rangle+\cdots \simeq \frac{c_{2}}{{x^{\prime}{ }_{12}^{2 \Delta_{\phi}}}_{12}}\left\langle\mathcal{A}\left(\vec{x}_{1}^{\prime}\right) \mathcal{A}\left(\vec{x}_{2}^{\prime}\right) \phi\left(\vec{x}_{3}^{\prime}\right)\right\rangle+\cdots
$$

Undoing the inversion operation and going to momentum space, we get the following expression

$$
\left\langle\phi_{\vec{k}_{1}} \phi_{\vec{k}_{2}} \phi_{\vec{k}_{3}}\right\rangle^{\prime}=c_{2} A_{0}^{i} A_{0}^{j}\left\langle A_{\vec{k}_{1}}^{i} A_{\vec{k}_{2}}^{j} \phi_{\overrightarrow{k_{3}}}\right\rangle^{\prime} \text {. }
$$

The three-point correlator on the right-hand side of the last expression is fixed by the Ward identities as found in Ref. [23] and reads

$$
\left\langle A_{\vec{k}_{1}}^{i} A_{\vec{k}_{2}}^{j} \phi_{\vec{k}_{3}}\right\rangle^{\prime}=\frac{c_{3}}{k_{1}^{3} k_{2}^{3}} \Pi^{i m}\left(k_{1}\right) \Pi^{m j}\left(k_{2}\right)=\frac{c_{3}}{k_{1}^{3} k_{2}^{3}}\left(\delta^{i j}-\hat{k}_{1}^{i} \hat{k}_{1}^{j}-\hat{k}_{2}^{i} \hat{k}_{2}^{j}+\hat{k}_{1} \cdot \hat{k}_{2} \hat{k}_{1}^{i} \hat{k}_{2}^{j}\right) .
$$

We finally find

$$
\left\langle\phi_{\vec{k}_{1}} \phi_{\vec{k}_{2}} \phi_{\vec{k}_{3}}\right\rangle^{\prime}=\frac{c_{\phi \phi \phi}}{k_{1}^{3} k_{2}^{3}}\left(1-\cos ^{2}\left(\hat{k}_{1} \cdot \hat{n}\right)-\cos ^{2}\left(\hat{k}_{2} \cdot \hat{n}\right)+\cos \left(\hat{k}_{1} \cdot \hat{k}_{2}\right) \cos \left(\hat{k}_{1} \cdot \hat{n}\right) \cos \left(\hat{k}_{2} \cdot \hat{n}\right)\right)+\operatorname{cyclic} .
$$

This expression again reproduces nicely the findings of Ref. [27].

\subsection{The higher-spin case}

The calculation of the three-point correlator from higher-spin states goes along the same lines of the previous section and we do not report here in full details. We just notice that one encounters permutations of the spin polarisation sum

$$
I_{s}\left(\vec{n}, \vec{k}_{1}, \vec{k}_{2}\right)=\left\langle A^{i_{1} \cdots i_{s}} A^{j_{1} \cdots j_{s}}\right\rangle \sum_{\lambda}\left\langle\epsilon_{i_{1} \cdots i_{s}}^{\lambda}\left(\vec{k}_{1}\right) \epsilon_{j_{1} \cdots j_{s}}^{* \lambda}\left(\vec{k}_{2}\right) \epsilon_{\ell_{1} \cdots \ell_{s}}^{\lambda}\left(\vec{k}_{1}\right) \epsilon_{\ell_{1} \cdots \ell_{s}}^{* \lambda}\left(\vec{k}_{2}\right)\right\rangle .
$$


Since the polarisation tensor is traceless we obtain

$$
I_{s}\left(\vec{n}, \vec{k}_{1}, \vec{k}_{2}\right)=A_{0}^{2} n^{i_{1}} \cdots n^{i_{s}} n^{j_{1}} \cdots n^{j_{s}} \sum_{\lambda} \epsilon_{i_{1} \cdots i_{s}}^{\lambda}\left(\vec{k}_{1}\right) \epsilon_{j_{1} \cdots j_{s}}^{* \lambda}\left(\vec{k}_{2}\right) \epsilon_{\ell_{1} \cdots \ell_{s}}^{\lambda}\left(\vec{k}_{1}\right) \epsilon_{\ell_{1} \cdots \ell_{s}}^{* \lambda}\left(\vec{k}_{2}\right) .
$$

To simplify this expression we average over the directions $n^{i}$ (an operation which is anyway done when comparing to the observations)

$$
I_{s, \text { av }}\left(\vec{k}_{1}, \vec{k}_{2}\right)=\int \mathrm{d} \Omega I_{s}\left(\vec{n}, \vec{k}_{1}, \vec{k}_{2}\right) .
$$

Using Eq. (3.9) we find that

$$
\begin{aligned}
I_{s, \mathrm{av}}\left(\vec{k}_{1}, \vec{k}_{2}\right) & =\int \mathrm{d} \Omega A_{0}^{2} n^{i_{1}} \cdots n^{i_{2 s}} \sum_{\lambda} \epsilon_{i_{1} \cdots i_{s}}^{\lambda}\left(\vec{k}_{1}\right) \epsilon_{i_{s+1} \cdots i_{2 s}}^{* \lambda}\left(\vec{k}_{2}\right) \epsilon_{\ell_{1} \cdots \ell_{s}}^{\lambda}\left(\vec{k}_{1}\right) \epsilon_{\ell_{1} \cdots \ell_{s}}^{* \lambda}\left(\vec{k}_{2}\right) \\
& =A_{0}^{2} \sum_{\lambda} \epsilon_{i_{1} \cdots i_{s}}^{\lambda}\left(\vec{k}_{1}\right) \epsilon_{i_{s}+1}^{* \lambda} \cdots i_{2 s}
\end{aligned}
$$

Using the normalization

$$
\int \mathrm{d} \Omega n^{i} n^{j}=\frac{4}{3} \delta^{i j}
$$

we find, after some combinatorics,

$$
I_{s, \text { av }}\left(\vec{k}_{1}, \vec{k}_{2}\right)=4 A_{0}^{2} \frac{s !}{(2 s+1) ! !} \sum_{\lambda}\left(\epsilon_{i_{1} \cdots i_{s}}^{\lambda}\left(\vec{k}_{1}\right) \epsilon_{i_{1} \cdots i_{s}}^{* \lambda}\left(\vec{k}_{2}\right)\right)^{2} .
$$

To calculate, for instance, the sum over helicities for $s=1$, we may use a coordinate system where the polarisation $\epsilon^{+}\left(\vec{k}_{1}\right)$ and $\epsilon^{+}\left(\vec{k}_{2}\right)$ are aligned. In this system, it is easy to find that

$$
\sum_{\lambda}\left(\epsilon_{i}^{\lambda}\left(\vec{k}_{1}\right) \epsilon_{i}^{* \lambda}\left(\vec{k}_{2}\right)\right)^{2}=1+\cos ^{2}\left(\hat{k}_{1} \cdot \hat{k}_{2}\right)
$$

which of course reproduces the direction average of the angle dependence in the expression (3.7). For a generic spin $s$ we find

$$
\sum_{\lambda}\left(\epsilon_{i_{1} \cdots i_{s}}^{\lambda}\left(\vec{k}_{1}\right) \epsilon_{i_{1} \cdots i_{s}}^{* \lambda}\left(\vec{k}_{2}\right)\right)^{2}=1+\cos ^{2 s}\left(\hat{k}_{1} \cdot \hat{k}_{2}\right)
$$

and therefore

$$
I_{s, \text { av }}\left(\vec{k}_{1}, \vec{k}_{2}\right)=4 A_{0}^{2} \frac{s !}{(2 s+1) ! !}\left(1+\cos ^{2 s}\left(\hat{k}_{1} \cdot \hat{k}_{2}\right)\right) .
$$

The averaged three-point correlator in the presence of higher-spin fields will be therefore of the form

$$
\left\langle\phi_{\vec{k}_{1}} \phi_{\vec{k}_{2}} \phi_{\vec{k}_{3}}\right\rangle_{\mathrm{av}}^{\prime}=\frac{c_{\phi \phi \phi}}{k_{1}^{3} k_{2}^{3}}\left(1+\cos ^{2 s}\left(\hat{k}_{1} \cdot \hat{k}_{2}\right)\right)+\text { cyclic. }
$$




\subsection{The three-point correlator involving higher-spin fields}

We want to use the OPE to write the three-point function $\left\langle A_{\vec{k}_{1}}^{i_{1} \cdots i_{s}} \phi_{\vec{k}_{2}} \phi_{\vec{k}_{3}}\right\rangle$. Using the inversion in the coordinates space, the OPE for a couple of scalar operators will have the form:

$$
\phi\left(\vec{x}_{2}^{\prime}\right) \phi\left(\vec{x}_{3}^{\prime}\right) \simeq \frac{1}{x_{23}^{\prime 2 \Delta_{\phi}}}\left(c_{0}+c_{1} \mathcal{A}\left(\vec{x}_{3}^{\prime}\right)+\cdots\right) \simeq \frac{1}{x_{23}^{\prime 2 \Delta_{\phi}}}\left(c_{0}+c_{1} A_{0}^{j_{1} \cdots j_{s}} A_{j_{1} \cdots j_{s}}\left(\vec{x}_{3}^{\prime}\right)+\cdots\right) .
$$

Thus, we can write

$$
\left\langle A^{i_{1} \cdots i_{s}}\left(\vec{x}_{1}^{\prime}\right) \phi\left(\vec{x}_{2}^{\prime}\right) \phi\left(\vec{x}_{3}^{\prime}\right)\right\rangle=\frac{c_{1}}{x_{23}^{\prime 2 \Delta_{\phi}}} A_{0}^{j_{1} \cdots j_{s}}\left\langle A^{i_{1} \cdots i_{s}}\left(\vec{x}_{1}^{\prime}\right) A_{j_{1} \cdots j_{s}}\left(\vec{x}_{3}^{\prime}\right)\right\rangle,
$$

which, performing the inversion backwards and transforming to the momentum space, becomes

$$
\begin{aligned}
\left\langle A_{\vec{k}_{1}}^{i_{1} \cdots i_{s}} \phi_{\vec{k}_{2}} \phi_{\vec{k}_{3}}\right\rangle^{\prime} & =\frac{c_{2}}{k_{2}^{3}} A_{0}^{j_{1} \cdots j_{s}}\left\langle A^{i_{1} \cdots i_{s}}\left(\vec{k}_{1}\right) A_{j_{1} \cdots j_{s}}\left(-\vec{k}_{1}\right)\right\rangle \\
& =\frac{c_{3}}{k_{1}^{3} k_{2}^{3}} A_{0}^{j_{1} \cdots j_{s}} \sum_{\lambda} \epsilon_{\lambda}^{i_{1} \cdots i_{s}}\left(\vec{k}_{1}\right) \epsilon_{\lambda j_{1} \cdots j_{s}}^{*}\left(\vec{k}_{1}\right)+\text { cyclic. }
\end{aligned}
$$

In the last step leading to (3.20) we have used the Ward identity associated to dilation isometry and the fact that the tensorial structure of the two higher-spin two-point function is given by the projector tensor $\Pi_{j_{1} \cdots j_{s}}^{i_{1} \cdots i_{s}}(k)$. Now we assume that the background vacuum expectation value of the higher-spin field has the usual structure in terms of one unit vector $\vec{n}$ and we get

$$
\left\langle A_{\vec{k}_{1}}^{i_{1} \cdots i_{s}} \phi_{\vec{k}_{2}} \phi_{\vec{k}_{3}}\right\rangle^{\prime}=\frac{c_{A \phi \phi}}{k_{1}^{3} k_{2}^{3}}\left(n^{j_{1}} \cdots n^{j_{s}} \Pi_{j_{1} \cdots j_{s}}^{i_{1} \cdots i_{s}}\left(k_{1}\right)\right)+\text { cyclic. }
$$

In the simplified case in which we consider $s=2$, we can expand the tensorial structure of (3.21)

$$
\begin{aligned}
n^{i} n^{j} \Pi_{i j}^{k l}\left(k_{1}\right) & =n^{i} n^{j}\left[\frac{1}{2}\left(\Pi_{i}^{k} \Pi_{j}^{l}+\Pi_{i}^{l} \Pi_{j}^{k}\right)-\frac{1}{2} \Pi_{i j} \Pi^{k l}\right]= \\
= & v^{k}\left(\hat{k}_{1}, \hat{n}\right) v^{l}\left(\hat{k}_{1}, \hat{n}\right)-\frac{1}{2} \sin ^{2}\left(\hat{k}_{1} \cdot \hat{n}\right) \Pi^{k l}\left(k_{1}\right),
\end{aligned}
$$

where

$$
v^{k}(\hat{k}, \hat{n})=\hat{n}^{k}-\hat{k}^{k} \cos (\hat{k} \cdot \hat{n})
$$

\subsection{The statistically anisotropic three-point correlator tensor-scalar- scalar from spinning particles}

Using the OPE we can also work out the statistically anisotropic contribution to the three-point correlator $\langle\gamma \phi \phi\rangle$ involving the massless spin-2 graviton state. We start from Eq. (3.4) to get

$$
\left\langle\phi\left(\vec{x}_{1}^{\prime}\right) \phi\left(\vec{x}_{2}^{\prime}\right) \gamma^{i j}\left(\vec{x}_{3}^{\prime}\right)\right\rangle \simeq \frac{c_{2}}{x_{12}^{\prime \Delta_{\phi}}}\left\langle\mathcal{A}^{2}\left(\vec{x}_{2}^{\prime}\right) \gamma^{i j}\left(\vec{x}_{3}^{\prime}\right)\right\rangle+\cdots \simeq \frac{c_{2}}{x_{12}^{\prime \Delta_{\phi}}}\left\langle\mathcal{A}\left(\vec{x}_{1}^{\prime}\right) \mathcal{A}\left(\vec{x}_{2}^{\prime}\right) \gamma^{i j}\left(\vec{x}_{3}^{\prime}\right)\right\rangle+\cdots
$$

Undoing the inversion operation and going to momentum space, we obtain

$$
\left\langle\phi_{\vec{k}_{1}} \phi_{\vec{k}_{2}} \gamma_{\overrightarrow{k_{3}}}^{i j}\right\rangle^{\prime}=c_{2} A_{0}^{i_{1} \cdots i_{s}} A_{0}^{j_{1} \cdots j_{s}}\left\langle A_{\vec{k}_{1}}^{i_{1} \cdots i_{s}} A_{\vec{k}_{2}}^{j_{1} \cdots j_{s}} \gamma i j_{\overrightarrow{k_{3}}}\right\rangle^{\prime} .
$$


For instance, for the spin-1 case, using the same techniques of Ref. [27] we get that the statistical anisotropic contribution reads

$$
\left\langle\phi_{\vec{k}_{1}} \phi_{\vec{k}_{2}} \gamma_{\overrightarrow{k_{3}}}^{\lambda}\right\rangle^{\prime} \supset \frac{c_{\phi \phi \gamma}}{k_{2}^{3} k_{3}^{3}} \epsilon_{i j}^{\lambda}\left(\hat{k}_{3}\right) v^{i}\left(\hat{k}_{1}, \hat{n}\right) v^{j}\left(\hat{k}_{2}, \hat{n}\right)
$$

For higher-spin fields, this expression generalizes to

$$
\left\langle\phi_{\vec{k}_{1}} \phi_{\vec{k}_{2}} \gamma_{\overrightarrow{k_{3}}}^{\lambda}\right\rangle^{\prime} \supset \frac{c_{\phi \phi \gamma}}{k_{2}^{3} k_{3}^{3}} \epsilon_{i j}^{\lambda}\left(\hat{k}_{3}\right) v^{i i_{2} \cdots i_{s}}\left(\hat{k}_{1}, \hat{n}\right) v_{i_{2} \cdots i_{s}}^{j}\left(\hat{k}_{2}, \hat{n}\right)
$$

where

$$
v^{i_{1} \cdots i_{s}}(\hat{k}, \hat{n})=n^{j_{1}} \cdots n^{j_{s}} \prod_{j_{1} \cdots j_{s}}^{i_{1} \cdots i_{s}}(\hat{k})
$$

\subsection{The statistically anisotropic four-point correlator from spinning particles in the collapsed limit}

We may also consider the four-point scalar correlator in the collapsed limit, that is the configuration in real space where two pairs of points, say $\vec{x}_{1}, \vec{x}_{2}$ and $\vec{x}_{3}, \vec{x}_{4}$ are very far from each other. Let us therefore consider the OPE expansion (3.18) as well as the one for the other (34) channel at the coincident point to get

$$
\left\langle\phi\left(\vec{x}_{1}\right) \phi\left(\vec{x}_{2}\right) \phi\left(\vec{x}_{3}\right) \phi\left(\vec{x}_{4}\right)\right\rangle \simeq \frac{1}{x_{12}^{2 \Delta_{\phi}} x_{34}^{2 \Delta_{\phi}}}\left(c_{0}+c_{1} A_{0}^{i_{1} \cdots i_{s}} A_{0}^{j_{1} \cdots j_{s}}\left\langle A_{i_{1} \cdots i_{s}}\left(\vec{x}_{1}\right) A_{j_{1} \cdots j_{s}}\left(\vec{x}_{3}\right)\right\rangle\right)+\cdots .
$$

The statistically anisotropic contribution to the four-point correlator therefore reads in momentum space with $\Delta_{\phi} \simeq 0$

$$
\left\langle\phi_{\vec{k}_{1}} \phi_{\vec{k}_{2}} \phi_{\vec{k}_{3}} \phi_{\vec{k}_{4}}\right\rangle^{\prime} \supset \frac{A_{0}^{i_{1} \cdots i_{s}} A_{0}^{j_{1} \cdots j_{s}}}{k_{2}^{3} k_{4}^{3}}\left\langle A_{i_{1} \cdots i_{s}}\left(\vec{k}_{12}\right) A_{j_{1} \cdots j_{s}}\left(-\vec{k}_{12}\right)\right\rangle+\operatorname{cyclic}, \quad\left(\vec{k}_{12}=\vec{k}_{1}+\vec{k}_{2} \simeq \overrightarrow{0}\right) \text {. }
$$

Assuming again the background for the higher-spin to be determined by a single vector $\vec{n}$, , we find then

$$
\left\langle\phi_{\vec{k}_{1}} \phi_{\vec{k}_{2}} \phi_{\vec{k}_{3}} \phi_{\vec{k}_{4}}\right\rangle^{\prime} \supset \frac{c_{\phi \phi \phi \phi} \sin ^{2 s}\left(\hat{k}_{12} \cdot \hat{n}\right)}{k_{12}^{3} k_{2}^{3} k_{4}^{3}}+\operatorname{cyclic}, \quad\left(\vec{k}_{12}=\vec{k}_{1}+\vec{k}_{2} \simeq \overrightarrow{0}\right) \text {. }
$$

\section{The statistically anisotropic correlators from partial higher-spin massless states}

Massless particles of spin $s$ in four-dimensional Minkowski space-time possess only helicities $\pm s$. On the other hand, massive fields have helicities belonging to the set $-s,-s+1, \ldots, s-1, s$. Analogously, in de Sitter space-time massive fields are allowed, although there exist additional fields, named "partially massless" fields [13-18], which do not belong to the aforementioned categories. In particular, fields with mass

$$
m^{2}=H^{2}[s(s-1)-p(p+1)]
$$


have helicities $-s,-s+1, \ldots, s-1, s$ where the helicities $-p,-p+1, \ldots, p-1, p$ have been removed for any $p \leq s-2$. Fields of this nature are described by totally symmetric tensors $A_{\mu_{1} \ldots \mu_{s}}$ whose linear action is invariant under the gauge transformation

$$
\delta A_{\mu_{1} \cdots \mu_{s}}=D_{\mu_{1}} \cdots D_{\mu_{s-p}} \xi_{\mu_{s-p+1} \cdots \mu_{s}}+\cdots,
$$

where $D_{\mu}$ is the covariant derivative, $\xi_{\mu_{s-p+1} \cdots \mu_{s}}$ is the gauge parameter and the ellipsis stands for further terms coming from the symmetrization of indices and contributions of terms with a number of derivatives fewer than $s-p-1$.

It is straightforward to notice that spin-1 fields in de Sitter do not possess partially massless states. The usual vector gauge invariance

$$
\delta A_{\mu}=D_{\mu} \xi
$$

can be acquired only be setting $m^{2}=0$. The first non-trivial case is the spin-2 field for which partial massless stases having four degrees of freedom exists with $p=0$ and $m^{2}=2 H^{2}$. The associated gauge invariance can be written explicitly as [18]

$$
\delta A_{\mu \nu}=D_{\mu} D_{\nu} \xi+H^{2} g_{\mu \nu} \xi
$$

Using the $\mathrm{dS} / \mathrm{CFT}_{3}$ correspondence, as a massless spin- $s$ field has its correspondent rank- $s$ conserved symmetric tensor in the boundary theory [18], the partially massless fields $A_{\mu_{1} \ldots \mu_{s}}$ correspond to partially conserved currents $L^{\mu_{1} \ldots \mu_{s}}$ on the boundary. In other words, since the coupling

$$
\int_{\tau=0} L^{\mu_{1} \cdots \mu_{s}} A_{\mu_{1} \cdots \mu_{s}}
$$

is gauge invariant, by applying the transformation to the higher-spin field and through straightforward manipulations, the following condition must be required on the currents $L^{\mu_{1} \ldots \mu_{s}}$

$$
D_{\mu_{1}} \cdots D_{\mu_{s-p}} L^{\mu_{1} \cdots \mu_{s}}=0
$$

Terms of lower order in derivatives which are proportional to the curvature of the boundary have been neglected. Using the fact that the boundary at $\tau \rightarrow 0$ is flat, one can interpret covariant derivatives in Eq. (4.6) as ordinary derivatives

$$
\partial_{i_{1}} \cdots \partial_{i_{s-p}} L^{i_{1} \cdots i_{s}}=0
$$

This condition can be used to characterize better the partial massless states. Notice that the dual field $L^{i_{1} \cdots i_{s}}$ is sourced by the boundary value of the corresponding higher-spin field $A_{i_{1} \cdots i_{s}}(\vec{x})$ and therefore the latter satisfies the same gauge transformation

$$
\delta A_{i_{1} \cdots i_{s}}(\vec{x})=D_{i_{1}} \cdots D_{i_{s-p}} \xi_{i_{s-p+1} \cdots i_{s}}+\cdots .
$$

Let us enter in more detail about the conformal algebra. It is well known that the conformal group $\mathrm{SO}(1,4)$ has ten generators, namely translations $\mathscr{P}_{i}$, dilations $\mathscr{D}$, special conformal transformations $\mathscr{K}_{i}$ and space rotations $\mathscr{L}_{i j}$. The corresponding conformal algebra is defined by the following commutation 
rules

$$
\begin{aligned}
& {\left[\mathscr{D}_{,} \mathscr{P}_{i}\right]=i \mathscr{P}_{i},} \\
& {\left[\mathscr{D}, \mathscr{K}_{i}\right]=-i \mathscr{K}_{i},} \\
& {\left[\mathscr{K}_{i}, \mathscr{P}_{j}\right]=2 i\left(\delta_{i j} \mathscr{D}-\mathscr{L}_{i j}\right),} \\
& {\left[\mathscr{L}_{i j}, \mathscr{P}_{k}\right]=i\left(\delta_{j k} \mathscr{P}_{i}-\delta_{i k} \mathscr{P}_{j}\right),} \\
& {\left[\mathscr{L}_{i j}, \mathscr{K}_{k}\right]=i\left(\delta_{j k} \mathscr{K}_{i}-\delta_{i k} \mathscr{K}_{j}\right),} \\
& {\left[\mathscr{L}_{i j}, \mathscr{D}\right]=0,} \\
& {\left[\mathscr{L}_{i j}, \mathscr{L}_{k l}\right]=i\left(\delta_{i l} \mathscr{L}_{j k}-\delta_{i k} \mathscr{L}_{j l}+\delta_{j k} \mathscr{L}_{i l}-\delta_{j l} \mathscr{L}_{i k}\right) .}
\end{aligned}
$$

Due to the properties of the algebra, it is useful to label the irreducible representations in terms of their scaling dimension $\Delta$ and their spin $s$ taking advantage of the commutativity of the generators $\mathscr{D}$ and $\mathscr{L}_{i j}$

$$
\begin{array}{r}
\mathscr{D}|\Delta, s\rangle=-i \Delta|\Delta, s\rangle, \\
\mathscr{L}_{i j}|\Delta, s\rangle_{l}=\left(\Sigma_{i j}\right)_{l}^{l^{\prime}}|\Delta, s\rangle_{l^{\prime}}
\end{array}
$$

Moreover, one can think of the operators $\mathscr{P}_{i}$ and $\mathscr{K}_{i}$ as being the raising and lowering (ladder) operators with respect to the scaling dimension. Indeed, using the algebra one finds:

$$
\begin{gathered}
\mathscr{D} \mathscr{P}_{i}|\Delta, s\rangle=i(\Delta+1) \mathscr{P}_{i}|\Delta, s\rangle, \\
\mathscr{D} \mathscr{K}_{i}|\Delta, s\rangle=i(\Delta-1) \mathscr{K}_{i}|\Delta, s\rangle .
\end{gathered}
$$

In what follows we also use the property for which, in radial quantization, $\mathscr{P}_{i}^{\dagger}=\mathscr{K}_{i}$. Taking advantage of the operator-state correspondence of the $\mathrm{CFT}_{3}$ which relates the operator $L^{\mu_{1} \cdots \mu_{s}}$ to a state $\left|L^{\mu_{1} \cdots \mu_{s}}\right\rangle$, we can analyse condition (4.6) further [18]. For instance, we already argued that for spin-1 condition (4.3) for the bulk field becomes $\partial_{i} L^{i}=0$ for the boundary field. Using the realization of the translation operator $\mathscr{P}_{i}$, we observe that the first descendant state of $\left|L^{i}\right\rangle$, which is $\mathscr{P}_{i}\left|L^{i}\right\rangle$, is required to be a null vector. Explicitly, one finds

$$
\begin{aligned}
\| \mathscr{P}_{i}\left|L^{i}\right\rangle \|^{2} & =\left\langle L^{r}\left|\mathscr{K}_{r} \mathscr{P}_{i}\right| L^{i}\right\rangle \\
& =\left\langle L^{r}\left|\left[\mathscr{K}_{r}, \mathscr{P}_{i}\right]\right| L^{i}\right\rangle \\
& =(h-2)\left\langle L^{i} \mid L_{i}\right\rangle=0,
\end{aligned}
$$

which constraints the conformal dimension of $L^{i}$ to be $h=2$. Going to the case of generic spins- $s$, due to the fact that $L^{i_{1} \cdots i_{s}}$ is partially conserved as in Eq. (4.6), the corresponding descendant must have vanishing norm

$$
\begin{aligned}
\| \mathscr{P}_{i_{1}} \mathscr{P}_{i_{2}} \cdots \mathscr{P}_{i_{m}}\left|L^{i_{1} i_{2} \cdots i_{s}}\right\rangle \|^{2} & =\left\langle L^{j_{1} j_{2} \cdots j_{s}}\left|\mathscr{K}_{j_{1}} \mathscr{K}_{j_{2}} \cdots \mathscr{K}_{j_{s}} \mathscr{P}_{i_{1}} \mathscr{P}_{i_{2}} \cdots \mathscr{P}_{i_{s}}\right| L^{i_{1} i_{2} \cdots i_{s}}\right\rangle \\
& \left.\sim m !(h-(s+1))(h-s) \cdots(h-(2+s-m)) \| L^{i_{1} i_{2} \cdots i_{s}}\right\rangle \|^{2} .
\end{aligned}
$$


In particular, we want to impose that the $m^{\text {th }}$-descendant with $m=r$ to be null, but nonvanishing if $m<r$ in order to match condition (4.6). This is achieved if

$$
h=2+s-r .
$$

Therefore, we can conclude that partially massless spin- $s$ fields in four dimensional de Sitter spacetime correspond to states on the boundary theory with conformal dimension given by condition (4.22), where $r$ ranges over the set $2, \cdots, s$.

Now, for $s>2$ partial massless states always exist for which $\Delta=0$. To show this we use the generic relation (1.1) between masses, Hubble rate and scaling dimensions. On the boundary $\tau=0$, the dominant scaling dimension for the partial massless states becomes

$$
\Delta=\frac{3}{2}-\sqrt{\left(s-\frac{1}{2}\right)^{2}-s(s-1)+p(p+1)}=\frac{3}{2}-\sqrt{\frac{1}{4}+p(p+1)} .
$$

For the choice

$$
p(p+1)=2 \Rightarrow p=1 \Rightarrow m^{2}=H^{2}[s(s-1)-2] \text { for } s>2,
$$

one gets $\Delta=0$ and since $p \leq s-2$, such state for which $p=1$ always exists. For instance, the case $s=3$ possesses two partially massless states, one for $p=1$ and $\Delta=0$ and the other for $p=0$ and $\Delta=1$. In Eq. (4.21) they correspond to $m=s-p=2$ and $m=s-p=3$, respectively. For $m=r=2$, one has $h=2+3-2=3$, corresponding to $\Delta=0$; for $m=r=3$, one has $h=2+3-3=2$, corresponding to $\Delta=1$. These values reproduce the conformal weights of the partially massless states. Of course we are interested in those partially massless states for which $\Delta=0$ as their corresponding fluctuations remain constant on super-Hubble scales.

For $s>3$, there exists always helicity states for which the corresponding $\Delta<0$. It is not clear if this is a problem as one should deal in any case with gauge-invariant quantities. For instance, due to the gauge transformation, $A_{\mu \nu}$ is not observable and one should construct a gauge-invariant tensor. Such off-shell tensor in de Sitter space is [35]

$$
\mathscr{F}_{\mu \nu \rho}=D_{\mu} A_{\nu \rho}-D_{\nu} A_{\mu \rho} .
$$

Therefore gauge-invariant quantities will contain in principle a sufficient number of derivatives to cancel the bad behaviour of the non gauge-invariant fields.

\subsection{The two-, three- and four-point correlators from partial massless particles}

We work with the partially massless states, which provide the representation of de Sitter isometry group and coincide with those of $\mathrm{CFT}_{3}$ on super-Hubble scales. We assume there is a mixing between the scalar field $\phi$ and the partially massless fields generated by a nonvanishing background of the higher-spin fields (so that our universe is a single realisation of the different ensembles) and by suitable couplings between the quadratic and cubic terms in the higher-spin fields and the scalar ${ }^{4}$. Alternatively,

\footnotetext{
${ }^{4}$ Explicit gauge-invariant cubic terms for partially massless fields have been constructed, in (A)dS in, for instance, Refs. [34,36,37].
} 
one can imagine to couple the scalar field to a gauge-invariant higher-spin field (a proper generalisation of $\mathscr{F}_{\mu \nu \rho}$ in Eq. (4.25)) which slightly away from exact de Sitter will contain the necessary couplings. On super-Hubble scales the partial masslessness of depth $n$ is defined by the condition ${ }^{5}$

$$
k^{i_{1}} \cdots k^{i_{n}} A_{i_{1} \cdots i_{s}}=0, \quad n \leq s .
$$

The condition (4.26) projects out the helicities $(-s+n,-s+n+1, \cdots, 0, \cdots, s-n-1, s-n)$ and therefore only the helicities $(-s, \cdots,-s+n-1, s-n+1, \cdots, s)$ are physical.

In the end, we are interested in partially massless spin- $s$ fields which posses a conformal dimension $\Delta=0$. The lowest possible spin is in fact $s=3$ with $p=1$. We provide the explicit construction for this particular case and then we generalise the results for partially massless field of spin $s$. In this case, the theory enjoys the gauge invariance

$$
A_{i j k}(k) \rightarrow A_{i j k}(k)+k_{i} k_{j} \xi_{k}(k)+\cdots
$$

since we are only interested in fields with scaling dimension $\Delta=0$. The contribution to the two-point function can be written as

$$
\phi_{\vec{k}} \phi_{-\vec{k}} \simeq \frac{c_{\phi}}{k^{3}}+c^{i_{1} i_{2} i_{3} j_{1} j_{2} j_{3}}(k) A_{i_{1} i_{2} i_{3}} A_{j_{1} j_{2} j_{3}}(0)+\cdots,
$$

where $c^{i_{1} i_{2} i_{3} j_{1} j_{2} j_{3}}$ is symmetric-traceless in $i_{1} i_{2} i_{3}$ and $j_{1} j_{2} j_{3}$ independently and satisfies

$$
c^{i_{1} i_{2} i_{3} j_{1} j_{2} j_{3}}(k) k_{i_{1}} k_{i_{2}} k_{j_{1}} k_{j_{2}}=0,
$$

due to gauge invariance, as well as being symmetric with respect to the exchange of the two groups of indices. Using Eq. (2.5), which implies $k_{i} \Pi^{i j}=0$, it is easy to verify that (4.29) is solved in terms of $\hat{k}_{i}$ and the projection tensor $\Pi^{i j}$ by

$$
\begin{aligned}
c^{i_{1} i_{2} i_{3} j_{1} j_{2} j_{3}}(k)= & P_{1}(k)\left[\hat{k}^{i_{1}} \hat{k}^{j_{1}}\left(\Pi^{i_{2} j_{2}} \Pi^{i_{3} j_{3}}+\Pi^{i_{2} j_{3}} \Pi^{i_{3} j_{2}}-\Pi^{i_{2} i_{3}} \Pi^{j_{2} j_{3}}\right)+\text { symm. }\right]+ \\
& P_{2}(k)\left(f^{i_{1} i_{2} i_{3} j_{1} j_{2} j_{3}}-\frac{1}{2} g^{i_{1} i_{2} i_{3} j_{1} j_{2} j_{3}}\right) .
\end{aligned}
$$

where $P_{1}(k)$ and $P_{2}(k)$ are two arbitrary functions to be fixed by dilation symmetry and in order to simplify the notation, we have defined

$$
\begin{aligned}
f^{i_{1} i_{2} i_{3} j_{1} j_{2} j_{3}} & =\Pi^{i_{1} j_{1}} \Pi^{i_{2} j_{2}} \Pi^{i_{3} j_{3}}+\Pi^{i_{1} j_{2}} \Pi^{i_{2} j_{1}} \Pi^{i_{3} j_{3}}+\Pi^{i_{1} j_{3}} \Pi^{i_{2} j_{1}} \Pi^{i_{3} j_{2}} \\
& +\Pi^{i_{1} j_{1}} \Pi^{i_{2} j_{3}} \Pi^{i_{3} j_{2}}+\Pi^{i_{1} j_{2}} \Pi^{i_{2} j_{3}} \Pi^{i_{3} j_{1}}+\Pi^{i_{1} j_{3}} \Pi^{i_{2} j_{2}} \Pi^{i_{3} j_{1}}
\end{aligned}
$$

and

$$
\begin{aligned}
g^{i_{1} i_{2} i_{3} j_{1} j_{2} j_{3}} & =\Pi^{i_{1} i_{2}} \Pi^{j_{1} j_{2}} \Pi^{i_{3} j_{3}}+\Pi^{i_{1} i_{3}} \Pi^{j_{1} j_{2}} \Pi^{i_{2} j_{3}}+\Pi^{i_{2} i_{3}} \Pi^{j_{1} j_{2}} \Pi^{i_{1} j_{3}} \\
& +\Pi^{i_{1} i_{2}} \Pi^{j_{1} j_{3}} \Pi^{i_{3} j_{2}}+\Pi^{i_{1} i_{3}} \Pi^{j_{1} j_{3}} \Pi^{i_{2} j_{2}}+\Pi^{i_{2} i_{3}} \Pi^{j_{1} j_{3}} \Pi^{i_{1} j_{2}} \\
& +\Pi^{i_{1} i_{2}} \Pi^{j_{2} j_{3}} \Pi^{i_{3} j_{1}}+\Pi^{i_{1} i_{3}} \Pi^{j_{2} j_{3}} \Pi^{i_{2} j_{1}}+\Pi^{i_{2} i_{3}} \Pi^{j_{2} j_{3}} \Pi^{i_{1} j_{1}} .
\end{aligned}
$$

It is easy to check that it is not possible to respect condition (4.29) with a term of the form $\hat{k}^{i_{1}} \hat{k}^{j_{1}} \hat{k}^{i_{2}} \hat{k}^{j_{2}} \Pi^{i_{3} j_{3}}$ as well as the term $\hat{k}^{i_{1}} \hat{k}^{j_{1}} \hat{k}^{i_{2}} \hat{k}^{j_{2}} \hat{k}^{i_{3}} \hat{k}^{j_{3}}$. Therefore, using

$$
\left\langle A_{i_{1} i_{2} i_{3}} A_{j_{1} j_{2} j_{3}}(0)\right\rangle=A_{0}^{2}\left[n_{i_{1}} n_{i_{2}} n_{i_{3}} n_{j_{1}} n_{j_{2}} n_{j_{3}}+\cdots\right]
$$

\footnotetext{
${ }^{5}$ The depth $n$ is defined to be $s-p$ where $p$ defines the missing helicities, see Eq. (4.1).
} 
we get

$$
\left\langle\phi_{\vec{k}} \phi_{-\vec{k}}\right\rangle^{\prime}=\frac{c_{\phi}}{k^{3}}\left[1+p_{1} \sin ^{6}(\hat{k} \cdot \hat{n})+p_{2} \sin ^{4}(\hat{k} \cdot \hat{n})\right] .
$$

Generalizing this result, the contribution from the partially massless higher-spin field of depth $n$ to the two-point function of a scalar field is

$$
\phi_{\vec{k}} \phi_{-\vec{k}}=\frac{c_{\phi}}{k^{3}}+c^{i_{1} \cdots i_{s} j_{1} \cdots j_{s}}(k) A_{i_{1} \cdots i_{s}} A_{j_{1} \cdots j_{s}}(0)+\cdots,
$$

where

$$
\begin{aligned}
c^{i_{1} \cdots i_{s} j_{1} \cdots j_{s}}(k) & =P_{1}(k)\left(\hat{k}^{i_{1}} \cdots \hat{k}^{i_{n-1}} \hat{k}^{j_{1}} \cdots \hat{k}^{j_{n-1}} \Pi^{i_{n} j_{n}} \cdots \Pi^{i_{s} j_{s}}+\cdots\right) \\
& +P_{2}(k)\left(\hat{k}^{i_{1}} \cdots \hat{k}^{i_{n-2}} \hat{k}^{j_{1}} \cdots \hat{k}^{j_{n-2}} \Pi^{i_{n-1} j_{n-1}} \cdots \Pi^{i_{s} j_{s}}+\cdots\right) \\
& +\vdots \\
& +P_{n}(k)\left(\Pi^{i_{1} j_{1}} \cdots \Pi^{i_{s} j_{s}}+\cdots\right) .
\end{aligned}
$$

The coefficients $P_{1}(k), \cdots, P_{n}(k)$ are straightforwardly fixed by the Ward identity from dilations. We indicate the vacuum expectation values of the partially massless higher fields by Eq. (2.25). Thus, we find

$$
\begin{aligned}
& \left\langle\phi_{\vec{k}} \phi_{-\vec{k}}\right\rangle^{\prime}=\frac{c_{\phi}}{k^{3}}\left[1+\tilde{p}_{1} \sin ^{2 s}(\hat{k} \cdot \hat{n})+\tilde{p}_{2} \sin ^{2(s-1)}(\hat{k} \cdot \hat{n}) \cos ^{2}(\hat{k} \cdot \hat{n})+\right. \\
& \left.\cdots+\tilde{p}_{n} \sin ^{2(s-n+1)}(\hat{k} \cdot \hat{n}) \cos ^{2(n-1)}(\hat{k} \cdot \hat{n})\right] \\
& =\frac{c_{\phi}}{k^{3}}\left[1+\sum_{m=0}^{n-1} p_{m+1} \sin ^{2(s-m)}(\hat{k} \cdot \hat{n})\right] .
\end{aligned}
$$

Finally, the contribution of the partial massless higher-spin fields to the averaged three-point function is given again by summing over the helicities $(-s, \cdots,-s+n+1, s-n-1, \cdots, s)$. The result for the contribution of partially massless higher-spin fields of depth $n$ turns out then to be

$$
\begin{aligned}
\left\langle\phi_{\vec{k}_{1}} \phi_{\vec{k}_{2}} \phi_{\vec{k}_{3}}\right\rangle_{\mathrm{av}}^{\prime} & =\frac{c_{\phi \phi \phi}}{k_{1}^{3} k_{2}^{3}}\left\{1+\cos ^{2 s}\left(\hat{k}_{1} \cdot \hat{k}_{2}\right)+p_{1} \cos \left(\hat{k}_{1} \cdot \hat{k}_{2}\right)\left[1+\cos ^{2 s-1}\left(\hat{k}_{1} \cdot \hat{k}_{2}\right)\right]+\cdots\right. \\
& \left.+p_{s-n+1} \cos ^{s-n+1}\left(\hat{k}_{1} \cdot \hat{k}_{2}\right)\left[1+\cos ^{s+n-1}\left(\hat{k}_{1} \cdot \hat{k}_{2}\right)\right]\right\}+ \text { cyclic } \\
& =\frac{c_{\phi \phi \phi}}{k_{1}^{3} k_{2}^{3}} \sum_{m=n-1}^{s} p_{s-m} \cos ^{s-m}\left(\hat{k}_{1} \cdot \hat{k}_{2}\right)\left[1+\cos ^{s+m}\left(\hat{k}_{1} \cdot \hat{k}_{2}\right)\right]+\text { cyclic }
\end{aligned}
$$

where $p_{0}=1$ and $p_{m}$ is the relative normalization of the polarization tensor of helicity $m$ to the higher helicity $s$.

Having determined the general formulas for the contribution of the partial massless higher-spin to the two- and three-point functions, it remains to specify the depth $n$. The latter can be specified from the requirement that the partial massless higher-spin field should be constant on super-Hubble scales, that is it should have scaling dimension $\Delta=0$. Using Eq. (1.1), the mass of the partially massless field of depth $n$ is

$$
m^{2}=H^{2}(n-1)(2 s-n) .
$$


Hence, the condition $\Delta=0$ gives $n=s-1$. In other words, partially massless higher-spin fields with spin $s$ and $\Delta=0$ have depth $n=s-1$ and polarizations $(-s, \cdots,-2,2, \cdots, s)$. Then, the contribution to the two- and three-point functions from the partially massless higher-spin fields are

$$
\left\langle\phi_{\vec{k}} \phi_{-\vec{k}}\right\rangle^{\prime}=\frac{c_{\phi}}{k^{3}}\left[1+\sum_{m=0}^{s-2} p_{m+1}^{\phi} \sin ^{2(s-m)}(\hat{k} \cdot \hat{n})\right]
$$

and

$$
\left\langle\phi_{\vec{k}_{1}} \phi_{\vec{k}_{2}} \phi_{\vec{k}_{3}}\right\rangle_{\mathrm{av}}^{\prime}=\frac{c_{\phi \phi \phi}}{k_{1}^{3} k_{2}^{3}} \sum_{m=0}^{s-2} p_{m}^{\phi \phi \phi} \cos ^{m}\left(\hat{k}_{1} \cdot \hat{k}_{2}\right)\left[1+\cos ^{2 s-m}\left(\hat{k}_{1} \cdot \hat{k}_{2}\right)\right]+\text { cyclic. }
$$

As for the four-point correlator in the collapsed limit, the statistically anisotropic contribution from the partially massless states reads

$$
\left\langle\phi_{\vec{k}_{1}} \phi_{\vec{k}_{2}} \phi_{\vec{k}_{3}} \phi_{\vec{k}_{4}}\right\rangle^{\prime} \supset \frac{c_{\phi \phi \phi \phi}}{k_{12}^{3} k_{2}^{3} k_{4}^{3}} \sum_{m=0}^{s-2} p_{m}^{\phi \phi \phi \phi} \sin ^{2(s-m)}\left(\hat{k}_{12} \cdot \hat{n}\right)+\text { cyclic, } \quad\left(\vec{k}_{12}=\vec{k}_{1}+\vec{k}_{2} \simeq \overrightarrow{0}\right) .
$$

We leave the relative coefficients $p_{m}$ among the various helicities free as in general one does not expect the respective vacuum expectation value to be related. If they are, the coefficients can be fixed using the special conformal transformations.

\section{Conclusions}

If light spinning particles exist during inflation, they leave a characteristic imprint on the scalar primordial cosmological perturbations. In this note we have investigated how the angle dependence of the statistical anisotropies should look like. We have been able to reproduce the well-known results of the spin-1 case and generalise it to higher-spin.

We conclude with a few comments. We have concentrated ourselves only on the calculation of the correlators of the scalar perturbations. Among the various correlators we have computed, there is the one involving higher-spin and scalar fluctuations. The significance of such correlators is not clear since, differently from the case of the standard massless spin-2 graviton, the higher-spin states are most probably doomed to decay and disappear after inflation. This will happen, for instance, for those massive higher-spin states which are rendered effectively massless only during inflation. Furthermore, one could investigate the possibility of the higher-spin field to play the role of the curvaton field and to be the ultimate responsible for the curvature perturbation.

In the case of partially massless higher-spin fields one will also require the construction of gaugeinvariant quantities for which correlation functions will not probably diverge in the infrared and also give physical significance to the $\Delta=0$ states, possibly by constructing generalized curvature terms for higher-spin fields [38]. The same will be required for higher-spin fields which acquire vanishing scaling dimension through couplings to matter. In such a case, as deduced from Ref. [24] these couplings will possibly involve mass terms an therefore they will not necessarily suffer of the same strong coupling problem of the corresponding spin- 1 case. Also, if couplings to matter will take place through gaugeinvariant quantities, one might worry about violating the necessary constraint which ensures that no ghost or extra states are propagating. In fact, one might violate the basic property that the constraints 
are only first order in time derivatives of the fields. In fact, as the new possible contributions will only involve dynamical matter fields, the unwanted time derivatives can be removed by making use of the matter field equations, see for instance Ref. [39].

Finally, there exist consistent theories of higher-spin four-dimensional gravity which come from the start with an infinite tower of massless higher-spin states [40]. They have been studied from the $\mathrm{dS} / \mathrm{CFT}_{3}$ correspondence point of view [41] and very recently a consistent characterisation of the Hilbert space of higher-spin quantum gravity in de Sitter has been proposed Ref. [42]. Interactions among the higher-spin states can be studied on the holographic dual side [43] and FRW-like solutions have been constructed in Ref. [44]. It is interesting to note that higher-spin fields with $s \geq 2$ do show the infrared phenomenon of accumulation of frozen modes and the consequent appearance of isotropy-breaking classical backgrounds. Higher-spin interactions with the standard massless graviton can also be a new source of gravitational waves from inflation.

These theories are possibly related to tensionless string theories. Strings have no tension and therefore their higher vibrational modes are unsuppressed, leading to an infinite tower of massless fields. Embedding this higher-spin theory into string theory and deforming the boundary field theory in a manner that turns on the bulk string tension will Higgs all of the higher-spin modes, giving them masses but leaving the bulk graviton massless [45]. It will be interesting to see if this phenomenon may deliver vanishing conformal weights and if it has some implication for inflation and its cosmological observables.

\section{Acknowledgments}

A.K. is partially supported by GGET project 71644/28.4.16. A.R. is supported by the Swiss National Science Foundation (SNSF), project Investigating the Nature of Dark Matter, project number: 200020159223. We thank the authors of Ref. [32] for interesting discussions and for comments on our draft.

\section{References}

[1] D. H. Lyth and A. Riotto, Phys. Rept. 314, 1 (1999) [hep-ph/9807278].

[2] X. Chen and Y. Wang, Phys. Rev. D 81, 063511 (2010) [astro-ph.Co/0909.0496].

[3] X. Chen and Y. Wang, JCAP 1004, 027 (2010) [hep-th/0911.3380].

[4] N. Arkani-Hamed and J. Maldacena, [hep-th/1503.08043].

[5] H. Lee, D. Baumann and G. L. Pimentel, JHEP 1612, 040 (2016) [hep-th/1607.03735].

[6] P. D. Meerburg, M. Mnchmeyer, J. B. Muoz and X. Chen, JCAP 1703, no. 03, 050 (2017) [astro-ph.C0/1610.06559].

[7] A. Moradinezhad Dizgah and C. Dvorkin, [astro-ph.C0/1708.06473].

[8] A. Strominger, JHEP 0110, 034 (2001) [hep-th/0106113].

[9] D. H. Lyth and D. Wands, Phys. Lett. B 524, 5 (2002) [hep-ph/0110002]. 
[10] A. Higuchi, Nucl. Phys. B 282, 397 (1987).

[11] L. Bordin, P. Creminelli, M. Mirbabayi and J. Noreña, JCAP 1609, no. 09, 041 (2016) [astro-ph.C0/1605.08424].

[12] A. Kehagias and A. Riotto, Fortsch. Phys. 65 (2017) no.5, 1700023 [hep-th/1701.05462].

[13] S. Deser and R. I. Nepomechie, Phys. Lett. 132B, 321 (1983).

[14] S. Deser and R. I. Nepomechie, Annals Phys. 154, 396 (1984).

[15] S. Deser and A. Waldron, Phys. Rev. Lett. 87, 031601 (2001).

[16] S. Deser and A. Waldron, Nucl. Phys. B 607, 577 (2001) [hep-th/0103198].

[17] S. Deser and A. Waldron, Nucl. Phys. B 662, 379 (2003).

[18] L. Dolan, C. R. Nappi and E. Witten, JHEP 0110, 016 (2001) [hep-th/0109096].

[19] S. Deser and A. Waldron, Phys. Lett. B 603, 30 (2004) [hep-th/0408155].

[20] B. Ratra, Astrophys.J. 391, L1-L4 (1992).

[21] J. Martin and J. i. Yokoyama, JCAP 0801, 025 (2008) [astro-ph/0711.4307].

[22] V. Demozzi, V. Mukhanov and H. Rubinstein, JCAP 0908, 025 (2009) [astro-ph.C0/0907.1030].

[23] M. Biagetti, A. Kehagias, E. Morgante, H. Perrier and A. Riotto, JCAP 1307, 030 (2013) [astro-ph.C0/1304.7785].

[24] A. Kehagias and A. Riotto, JCAP 1707 (2017) no.07, 046 [hep-th/1705.05834].

[25] M. a. Watanabe, S. Kanno and J. Soda, Prog. Theor. Phys. 123, 1041 (2010) [astro-ph.C0/1003.0056].

[26] J. Soda, Class. Quant. Grav. 29, 083001 (2012) [hep-th/1201.6434].

[27] N. Bartolo, S. Matarrese, M. Peloso and A. Ricciardone, Phys. Rev. D 87, no. 2, 023504 (2013) [astro-ph.C0/1210.3257].

[28] N. Bartolo, A. Kehagias, M. Liguori, A. Riotto, M. Shiraishi and V. Tansella, Phys. Rev. D 97, no. 2, 023503 (2018) [astro-ph.C0/1709.05695].

[29] J. L. Cardy, Nucl. Phys. B 290 (1987) 355.

[30] A. Kehagias and A. Riotto, Nucl. Phys. B 864, 492 (2012) [hep-th/1205.1523].

[31] A. Kehagias and A. Riotto, Nucl. Phys. B 868, 577 (2013) [hep-th/1210.1918].

[32] D. Baumann, G. Goon, H. Lee and G. L. Pimentel, [hep-th/1712.06624].

[33] J. M. Maldacena, JHEP 0305, 013 (2003) [astro-ph/0210603]. 
[34] L. Apolo, S. F. Hassan and A. Lundkvist, Phys. Rev. D 94, no. 12, 124055 (2016) [hep-th/1609.09515].

[35] S. Deser and A. Waldron, Phys. Rev. D 74, 084036 (2006) [hep-th/0609113].

[36] E. Joung, L. Lopez and M. Taronna, JHEP 1207, 041 (2012) [hep-th/1203.6578].

[37] E. Joung, L. Lopez and M. Taronna, JHEP 1301, 168 (2013) [hep-th/arXiv:1211.5912].

[38] R. Manvelyan and W. Ruhl, Nucl. Phys. B 797, 371 (2008) [hep-th/0705.3528].

[39] S. Deser and A. Waldron, Phys. Rev. D 74, 084036 (2006) [hep-th/0609113].

[40] M. A. Vasiliev, Phys. Lett. B 243, 378 (1990).

[41] D. Anninos, T. Hartman and A. Strominger, Class. Quant. Grav. 34, no. 1, 015009 (2017) [hep-th/1108.5735].

[42] D.Anninos, F. Denef, R. Monten, and Z. Sun, [hep-th/1711.10037]

[43] C. Sleight, [hep-th/1610.01318].

[44] R. Aros, C. Iazeolla, J. Norea, E. Sezgin, P. Sundell and Y. Yin, [hep-th/1712.02401].

[45] M. R. Gaberdiel, C. Peng, and I. G. Zadeh, JHEP 10, 101 (2015) [hep-th/1506.02045]. 